\title{
Differentiating Scrub Typhus, Dengue Fever and Typhoid Fever in Indian Children Presenting with Acute Febrile Illness
}

\section{Saheli Misra Chatterjee, Suman Mondal, Kaushik Mukhopadhyay and Niloy Kumar Das}

Department of Paediatrics, Employees State Insurance Post Graduate Institute of Medical Sciences and Research and Employees State Insurance Corporation Medical College, Joka, Kolkata, West Bengal, India

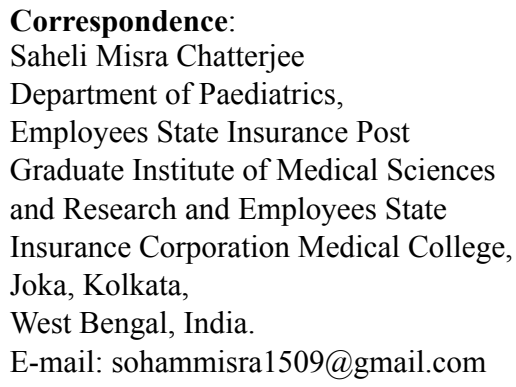

DOI: $10.3126 /$ jnps.v40i2.29319

Submitted on: $2020-06-6$

Accepted on: 2020-07-28

\section{Acknowledgements: None}

Funding: Nil

Conflict of Interest: None declared

Permission from IRB: Yes

To cite this article: Chatterjee SM, Mondal S, Mukhopadhyay K, Das NK. Differentiating scrub typhus, dengue fever and typhoid fever in Indian children presenting with acute febrile Illness. J Nepal Paediatr Soc. 2020;40(2):93-9.

\section{ABSTRACT}

Introduction: The disease spectrum of dengue, scrub typhus and typhoid presenting as acute febrile illness is often a diagnostic dilemma to the clinician. The purpose of this study is to compare the clinical features and laboratory parameters of children suffering from typhoid, dengue and scrub typhus and use these parameters in early identification of scrub typhus before conclusion is made from serological diagnosis.

Methods: A retrospective observational analytical study was conducted among children presenting with acute febrile illness in a tertiary care level hospital. Over the period of one year 113 cases were identified of which 39 were dengue, 44 were typhoid and 30 were scrub typhus.

Results: The mean age of the children was $7.45 \pm 2.98$ years, median was eight with an interquartile range of six to ten years. The male to female ratio was $1.3: 1$. Symptoms of vomiting $(61.54 \%)$, headache $(46.15 \%)$ and hepatomegaly $(47.37 \%)$ were significantly higher among children with dengue fever. A significantly higher number of children with scrub typhus fever had temperature above $40^{\circ} \mathrm{C}$. The incidence of low haemoglobin, raised C reactive protein, raised ALT and low albumin levels were significantly higher in them. Children with acute febrile illness having temperature $>40^{\circ} \mathrm{C}$, absolute neutrophil/lymphocyte ratio $>2$ early in the illness were more likely to suffer from scrub typhus with relative probability ratio (RPR) of 25.68 and 10.57 respectively $(\mathrm{p}<0.001)$. Children with WBC $<5000 / \mathrm{mm}^{3}$ were more likely to be suffering from dengue with RPR of $10.60(\mathrm{p}<0.001)$.

Conclusion: Children with acute febrile illness with temperature $>40^{\circ} \mathrm{C}$ and absolute neutrophil/lymphocyte ratio $>2$ early in the illness were more likely to be suffering from scrub typhus.

Keywords: acute febrile illness; dengue; scrub typhus; typhoid

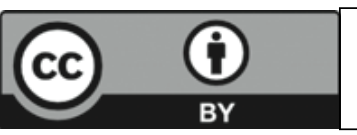

This work is licensed under creative common attribution 3.0 license 


\section{INTRODUCTION}

The burden of infection in our country is quite high and leads to high child mortality. Lately there has been resurgence of vector borne infections like dengue and scrub typhus. Scrub typhus is the most common re- emerging rickettsial infection in India and is being reported from southern districts of West Bengal and Eastern India. ${ }^{1-5}$ The disease is prevalent all over India. ${ }^{6}$ Studies in southern part of India report a seroprevalence of 15 to $40 \%$ in general population. ${ }^{7,8}$ In a multi-centric study of etiology of acute undifferentiated fever in India, $16 \%$ was diagnosed as dengue, $35 \%$ as salmonella infections and $10 \%$ as scrub typhus. Among the other illnesses were malaria $17 \%$, bacteraemia $8 \%$, leptospirosis 7\%, and chikungunya $6 \% .{ }^{9}$ In a similar study from southern India among adults dengue was $43.5 \%$ followed by enteric fever at $4.61 \%$ and scrub typhus at $0.15 \%$ among others. ${ }^{10}$ It is also noteworthy that $29.9 \%$ had undiagnosed fever. With the rise in incidence of scrub typhus it is difficult to differentiate the cause of acute febrile illness from typhoid and dengue due to similar presentations. Previous studies have reported on clinical scores to differentiate between dengue and scrub typhus. ${ }^{11,12}$ A study from Thailand in adults differentiating between scrub typhus and dengue reported increased association of hemorrhagic manifestation, low platelet and low white blood cell count with dengue. ${ }^{13}$ An eschar, which is supposed to indicate scrub typhus, is not always found. ${ }^{14}$ However studies differentiating scrub typhus from dengue are limited among paediatric patients and hence the following study was planned. The purpose of this study was to compare the clinical features, laboratory parameters in children suffering from typhoid, dengue and scrub typhus and determine whether the findings of scrub typhus were similar to those of typhoid fever and dengue fever and to determine any combination of clinical and laboratory investigations that will help in early identification of scrub typhus before serological diagnosis is available

\section{METHODS}

This is a prospective observational descriptive study conducted in the Paediatric ward of our hospital over a period of one year from August
2018 till August 2019. Research approval was taken from the institutional ethics committee. All children with acute febrile illness under 12 years admitted to the paediatric ward from the outpatient and emergency department were clinically assessed. Daily temperature was monitored in the ward and the associated symptoms and signs were recorded in the history sheets. Lymphadenopathy was documented if the size of nodes were greater than $2 \mathrm{~cm}$ in diameter. Hepatomegaly and splenomegaly were documented clinically and on ultrasound-based on organometric data. ${ }^{15}$ Blood smears and vacutainers of blood were collected from study participants to carry out diagnostic assays. Blood and urine culture were done as indicated to rule out other infections. Malaria was diagnosed using rapid diagnostic tests (RDT) and microscopy. Dengue, typhoid, and scrub typhus infections were identified using rapid diagnostic test kits and ELISA on or beyond five days of fever. Blood was also collected for assessment of their liver and kidney function. The inclusion criteria included children with positive serology for typhoid, scrub or dengue. We excluded children with co morbid conditions like congenital heart disease, thalassemia, asthma, etc. and suffering from dual infections. At discharge, when a definite diagnosis of the illness was established, the case details were recorded in the proforma maintaining confidentiality of record. Consent was taken from the caregiver of the child before discharge. Based on serological diagnosis they were divided into three groups.

Data was entered and analysed using SPSS version 22. The means, SD medians, interquartile range, frequencies and percentages were calculated for parametric and non- parametric variables. The Kruskal Wallis and ANOVA test was used to compare non-parametric and parametric continuous variables across three groups. The Fisher's exact and chi square test with crosstabs was used to compare categorical variables. Multivariate analysis was performed in STATA ${ }^{\circledR}$ v 16.1 using multinomial logistic regression analysis in which the relative risk/probability ratios of significant variables were compared among three groups. Statistical significance was set at $\mathrm{p}<0.05$. 

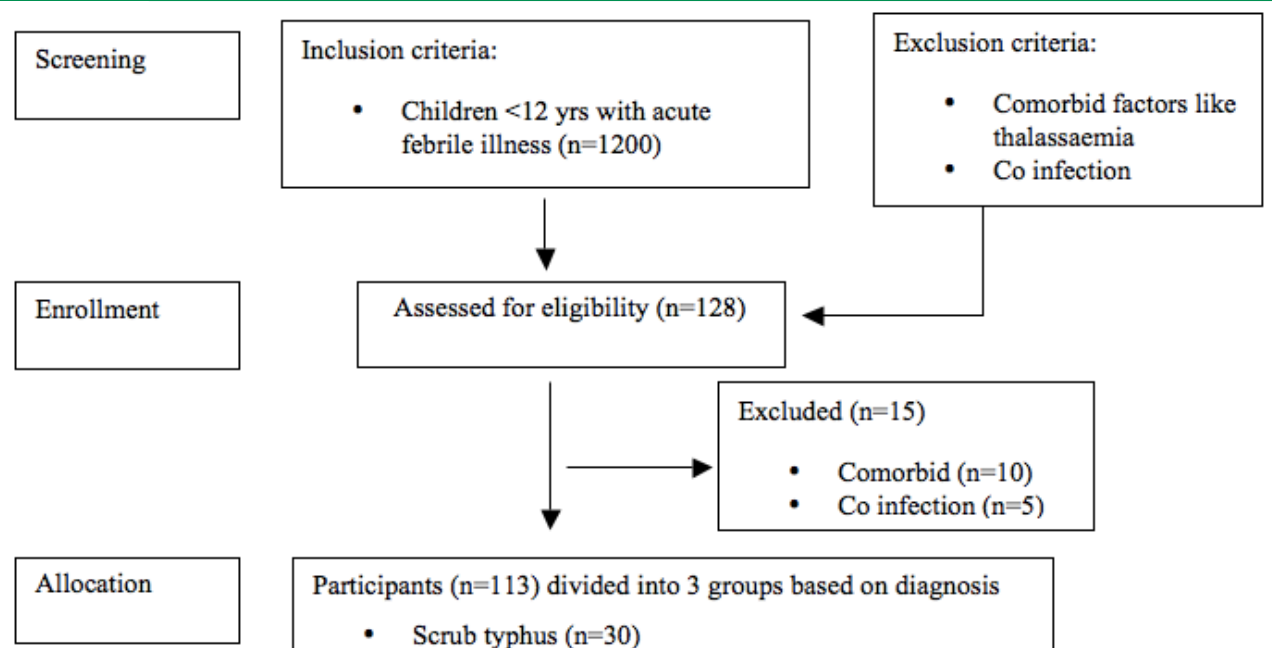

$$
\begin{aligned}
& \text { Participants }(\mathrm{n}=113) \text { divided into } 3 \text { groups based on diagnosis } \\
& \text { - Scrub typhus }(\mathrm{n}=30) \\
& \text { - } \text { Dengue }(\mathrm{n}=39) \\
& \text { - }
\end{aligned}
$$

Figure 1. Our prospective study flowchart

\section{RESULTS}

Twelve hundred children were admitted with acute febrile illness. Eighty-four patients were positive for malaria. One hundred and twenty-eight children tested positive for dengue, typhoid and scrub typhus by either RDT or ELISA or both tests.
Of them, ten children were excluded due to comorbid conditions. Five children tested positive for more than one infection. Out of remaining 113 children, 39 tested positive for dengue by either

\begin{tabular}{|c|c|c|c|c|c|}
\hline Clinical characteristics & $\begin{array}{l}\text { Typhoid fever } \\
\mathrm{N}=44\end{array}$ & $\begin{array}{l}\text { Scrub typhus } \\
\mathbf{N}=\mathbf{3 0}\end{array}$ & $\begin{array}{l}\text { Dengue } \\
\mathbf{N}=\mathbf{3 9}\end{array}$ & $X^{2}$ & p value \\
\hline Age in years, median (IQR) & $8(5 ; 9)$ & $8(6.5 ; 11)$ & $8(6 ; 10)$ & 4.61 & 0.1 \\
\hline Males, n (\%) & $28(63.64)$ & $18(60)$ & $18(46.15)$ & 2.76 & 0.25 \\
\hline $\begin{array}{l}\text { Fever duration before first visit in } \\
\text { days, median (IQR) }\end{array}$ & $5(5-6)$ & $6(5-7)$ & $4(3-5)$ & 36.62 & $<.001$ \\
\hline Cough, n (\%) & $14(31.82)$ & $8(26.6)$ & $3(7.69)$ & 7.47 & $<.05$ \\
\hline Skin rash, n (\%) & $9(20.45)$ & $10(33.3)$ & $4(10.26)$ & 5.49 & 0.06 \\
\hline Vomiting, n (\%) & $8(18.18)$ & $14(46.74)$ & $24(61.54)$ & 16.70 & $<.001$ \\
\hline Headache n (\%) & $2(4.55)$ & $12(40)$ & $18(46.15)$ & 20.37 & $<.001$ \\
\hline Loose stool n (\%) & $2(4.55)$ & $4(13.3)$ & $6(15.38)$ & 2.88 & 0.24 \\
\hline Constipation n (\%) & $12(27.3)$ & $4(13.3)$ & $2(7.69)$ & 6.48 & $<.05$ \\
\hline Abdominal pain $\mathrm{n}(\%)$ & $4(9.1)$ & $1(3.33)$ & $6(23.08)$ & 2.84 & 0.24 \\
\hline Cervical lymphadenopathy, n (\%) & $12(27.27)$ & $8(26.67)$ & $6(15.79)$ & 1.96 & 0.37 \\
\hline Hepatomegaly, n (\%) & $2(4.55)$ & $6(20)$ & $18(47.37)$ & 31.31 & $<.05$ \\
\hline Splenomegaly, n (\%) & $2(4.55)$ & $8(26.67)$ & $0(0)$ & 13.74 & $<.001$ \\
\hline Hepatosplenomegaly & $10(22.73)$ & $8(26.67)$ & $6(15.79)$ & 1.39 & 0.5 \\
\hline Body Temperature in ${ }^{\circ} \mathrm{C}$, mean (SD) & $39.24(0.84)$ & $39.68(0.64)$ & $39.1(0.9)$ & & $<.01$ \\
\hline Body Temperature $>40^{\circ} \mathrm{C}, \mathrm{n}(\%)$ & $4(9.1)$ & $16(53.3)$ & $7(17.95)$ & 20.36 & $<.001$ \\
\hline Fever duration in days median (IQR) & $6.00(6.00 ; 7.50)$ & $11.0(9.00 ; 11.0)$ & $5.00(5.00 ; 6.00)$ & 45.13 & $<0.001$ \\
\hline
\end{tabular}
RDT or ELISA, 44 tested positive for typhoid by ELISA specific for typhoid IgM. Thirty were

Table 1. Our prospective study flowchart 

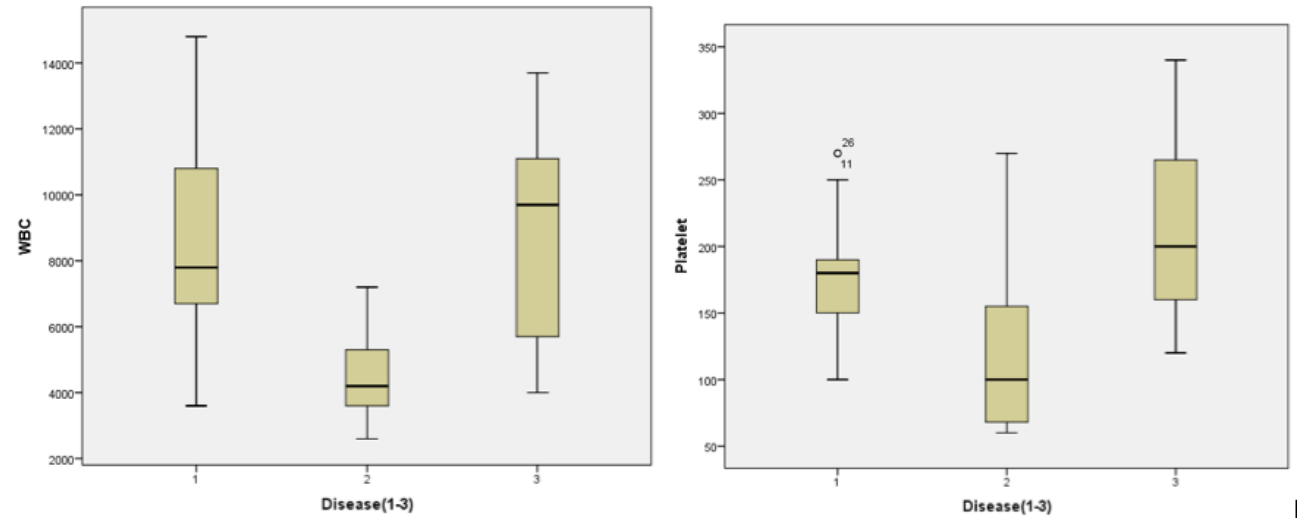

Figure 2. Box plot showing WBC and platelet count in scrub typhus (1), dengue (2) and typhoid fever (3)

positive for scrub typhus by ELISA specific for scrub typhus IgM.

The mean age of the children was $7.45 \pm 2.98$ years, median was eight with interquartile range of six to 10 years. The male to female ratio was $1.3: 1$. The children were grouped into three groups on the basis of their diagnosis: scrub typhus (30), dengue fever (39) and typhoid fever (44). The mean age of children with scrub typhus was $7.63 \pm 2.97$ years, typhoid was $6.85 \pm 2.86$ years and dengue was 8.15 \pm 2.75 years $(p<0.001)$. There was male preponderance across all diseases but was not statistically significant. The children with dengue sought medical care earlier than scrub or typhoid ( $p$ $<0.01)$. The duration of fever from onset till the day fever subsided was the most among children with scrub typhus who had a significantly longer duration of fever for 11 days compared to six days in typhoid and five days in dengue $(\mathrm{p}<0.05)$ as shown in Table 1.

There were certain features like eschar in eight $(28.5 \%)$, neck rigidity in six $(20 \%)$ and cranial

Table 2. Comparison of laboratory parameters in typhoid fever, scrub typhus and dengue

\begin{tabular}{llllr}
$\begin{array}{l}\text { Variables for laboratory } \\
\text { parameters }\end{array}$ & $\begin{array}{l}\text { Typhoid fever } \\
\text { N=44 }\end{array}$ & $\begin{array}{l}\text { Scrub typhus } \\
\text { N=30 }\end{array}$ & $\begin{array}{l}\text { Dengue } \\
\text { N=39 }\end{array}$ & p value \\
\hline $\begin{array}{l}\text { Duration from fever to CBC (days), } \\
\text { median (IQR) }\end{array}$ & $6(5 ; 7)$ & $7(6 ; 8)$ & $5(4 ; 5)$ & $<0.001$ \\
Hemoglobin gm/dl median (IQR) & $10.5(10.0 ; 11.8)$ & $10.4(9.90 ; 11.1)$ & $11.4(11.2 ; 12.5)$ & $<0.001$ \\
HCT \% median (IQR) & $31.5(30.0 ; 36.2)$ & $31.2(29.9 ; 33.0)$ & $35.4(33.6 ; 37.4)$ & $<0.001$ \\
WBC/mm ${ }^{3}$,median (IQR) & $7200(5700 ; 10400)$ & $7800(6200-10800)$ & $4200(3650 ; 5250)$ & $<0.001$ \\
ANC: ALC mean (SD) & $1.13(0.64)$ & $2.47(1.11)$ & $1.17(0.96)$ & $<0.001$ \\
Atypical lymphocytes $>5 \%, \mathrm{n}(\%)$ & $2(4.55)$ & $6(20)$ & $3(7.69)$ & 0.082 \\
PLT x103/mm 3 , median (IQR) & $200(160-265)$ & $180(100-270)$ & $100(68-155)$ & $<.001$ \\
PLT<100 x10 $/$ mm 3 ,n (\%) & $0(0)$ & $2(6.67)$ & $18(46.15)$ & 0.000 \\
BUN in mg/dl, median (IQR) & $19(13-25)$ & $22(21-26)$ & $22(19-29)$ & 0.46 \\
Cr in mg/dl, median (IQR) & $0.7(0.6-0.8)$ & $0.8(0.6-1)$ & $0.8(0.7-0.9)$ & 0.61 \\
AST in U/l, median (IQR) & $60(32-98)$ & $66(45-111)$ & $112(98-176)$ & 0.15 \\
ALT in U/l, median(IQR) & $22(14-46)$ & $64(39-86)$ & $73(30-126)$ & 0.04 \\
Albumin g/dl, median (IQR) & $4.50(4.50 ; 4.60)$ & $3.70(3.50 ; 3.70)$ & $4.25(4.10 ; 4.50)$ & $<.001$ \\
CRP m/dl median (IQR) & $36.5(5.74 ; 40.6)$ & $45.0(7.77 ; 59.0)$ & $8.50(7.30 ; 10.0)$ & 0.013
\end{tabular}

ANC: absolute neutrophil count; ALC: absolute lymphocyte count; PLT: Platelet; Cr: creatinine; AST: aspartate 
Table 3. Multinomial logistic regression model comparing scrub typhus fever and dengue with typhoid fever as reference/base category

\begin{tabular}{|llrrrrrr|}
\hline \multirow{2}{*}{ Variable } & Unit of change & \multicolumn{3}{c}{ Scrub typhus } & & \multicolumn{2}{c}{ Dengue } \\
\cline { 2 - 7 } & & RPR & CI (95\%) & p value & RPR & CI (95\%) & p value \\
\hline Body temperature & $\begin{array}{l}\mathrm{BT}<40 \mathrm{vs} \mathrm{BT}> \\
40^{\circ} \mathrm{C}\end{array}$ & 25.68 & $4.11-160.52$ & $<0.001$ & 2.12 & $0.33-13.46$ & 0.43 \\
WBC & $\begin{array}{l}<500 \mathrm{vs}>5000 / \\
\mathrm{mm}^{3}\end{array}$ & 0.73 & $0.08-6.42$ & 0.78 & 10.60 & $2.98-37.72$ & $<0.001$ \\
$\begin{array}{l}\text { Neutrophil/ } \\
\text { lymphocyte ratio } \\
\text { CRP }\end{array}$ & $2 \mathrm{vs}>2$ & 10.57 & $2.55-43.87$ & $<0.001$ & 1.11 & $0.27-4.58$ & 0.88 \\
& $<10 \mathrm{vs}>10 \mathrm{mg} / \mathrm{dl}$ & 0.44 & $0.11-1.77$ & 0.25 & 2.48 & $0.82-7.48$ & 0.11
\end{tabular}

nerve involvement in two $(6.67 \%)$ children with scrub typhus. Symptoms like vomiting (61.54\%), headache (46.15\%) and hepatomegaly (47.37\%) were significantly higher among children with dengue fever (Table 1). There was significant difference in the mean body temperature among different groups with significantly higher number of children with temperature $>40^{\circ} \mathrm{C}$ in scrub typhus fever. The incidence of hepatomegaly was significantly higher among children suffering from dengue. Hepatosplenomegaly though higher in scrub typhus was not statistically significant. Splenomegaly was not found in dengue.

The laboratory investigations suggest that haemoglobin concentration was significantly lower in scrub typhus and typhoid infection. Haematocrit was significantly higher in children with dengue (Table 2). The WBC count is lower in dengue than the other groups. The absolute neutrophilic count to absolute lymphocyte count ratio is significantly higher among children suffering from scrub typhus though the WBC count remains in the normal range. The median (IQR) platelet count was significantly lower among children suffering from dengue and $46.15 \%$ children reported to have platelet count of $<100 \times 10^{3} / \mathrm{mm}^{3}(\mathrm{p}<0.001)$. The median (IQR) of $\mathrm{C}$ reactive protein in children with scrub typhus was $45.0(7.77 ; 59.0)$ which was significantly higher than $36.5(5.74 ; 40.6)$ in typhoid and $8.50(7.30 ; 10.0)$ in dengue $(\mathrm{p}<0.05)$. The albumin level in scrub typhus was significantly lower than in the children with typhoid fever and dengue $(p=0.006)$. The transaminase levels were raised in all three groups with statistically significant rise in alanine amino transferase (ALT) in scrub typhus.

We highlighted the findings in Table 1 and 2 that suggested significance of $<0.05$ thus rejecting our above hypothesis that the findings in scrub typhus are similar to typhoid and dengue fever. Despite statistically significant differences many individual clinical or laboratory variables were poor discriminators between the diseases, early in the illness. We wanted to know whether the findings in scrub typhus will help in early diagnosis of the illness. Multinomial regression analysis was performed on only 100 out of 113 patients due to missing data. Children with acute febrile illness having temperature $>40^{\circ} \mathrm{C}$ absolute neutrophil/ lymphocyte ratio $>2$ early in the illness were more likely to suffer from scrub typhus with relative probability ratio (RPR) of 25.68 and 10.57 respectively $(\mathrm{p}<0.001)$. Children with $\mathrm{WBC}<$ $5000 / \mathrm{mm}^{3}$ were more likely to be suffering from dengue with RPR of $10.60(\mathrm{p}<0.001)$. (Table 3) Children with low CRP were more likely to have dengue and less likely to suffer from typhoid fever or scrub typhus but was not statistically significant.

\section{DISCUSSION}

This study was undertaken primarily to help us differentiate scrub typhus from typhoid and dengue till the serological diagnosis is awaited. Often specific signs of scrub like eschar are not visible. ${ }^{6,16,17}$ The dilemma differentiating typhus from typhoid dates back to 1853 since the publication in Boston surgical and medical journal when its presentation was thought to be two different manifestation of a single disease. ${ }^{18} \mathrm{We}$ 
noted that dengue fever is marked by significant vomiting and hepatomegaly previously reported in a comparative study of dengue with scrub typhus in Thai children, though they noted significant hepatomegaly with scrub typhus. ${ }^{19}$ The fact that none had splenomegaly has been reported in a study from South India that compares severe dengue with scrub typhus in children and states that almost ninety percent of children presented with significant hepatomegaly and none had splenomegaly. ${ }^{20}$

Low WBC and low platelet count seen in dengue fever has been reported by several studies but they were not able to significantly differentiate scrub typhus from dengue. ${ }^{13,20}$ All our cases except one of dengue were primary cases and none of them had bleeding manifestation. Only one of them suffered from hypotension. Haemoglobin and albumin level were significantly lower in scrub typhus as reported previously. ${ }^{21,22}$ The low serum albumin level though documented, but the timing of investigation in the illness varied. Moreover low serum albumin is an indication of severity of the illness due to complication $^{23}$ so its role in early diagnosis has to be further studied. The serum aspartate and alanine transaminase levels were raised across all three diseases as previously noted in studies. ${ }^{13,19} \mathrm{~A}$ significantly raised $\mathrm{C}$ reactive protein is found in scrub typhus which is however lower than the mean value of $76.05 \pm 68.416$ reported in an observational study from India. ${ }^{24}$ It was noted that CRP levels in scrub typhus at initial presentation was often low but would be high subsequently if left untreated explaining the wide IQR.

The clinical spectrum of the three diseases was not helpful in predicting scrub typhus except the high rise of temperature that was seen in these children and has been reported previously. ${ }^{19}$ We found that the presence of body temperature $>40^{\circ} \mathrm{C}$ and absolute neutrophil to lymphocyte ratio $>2$ increases the probability of suffering from scrub typhus by 25 and 10 folds respectively though the likelihood of increased body temperature with scrub typhus has been previously reported. ${ }^{19}$

Our study had many limitations as sample size was not adequate. The number of cases was limited in the one-year study period due to their seasonal presentation. The data in relation to kidney and liver function was not available in all cases and the timing of the investigation from its presentation should have been uniformly maintained. As it includes symptomatic children admitted to hospital for evaluation, the spectrum of these diseases that does not present to hospital or are outpatient attendees are not included. Hence potentially useful clinical variables may have been missed.

\section{CONCLUSIONS}

With increased awareness of scrub typhus and its clinical and laboratory parameters we may be able to discriminate this disease from typhoid and dengue early in illness in resource limited settings. We note that a high temperature above $40^{\circ} \mathrm{C}$ and a neutrophil/lymphocyte ratio $>2$ in the presence of a normal white cell count may increase the chances of scrub typhus infection in a tropical setting of frequent typhoid and dengue infection.

\section{REFERENCES}

1. Dass R, Deka NM, Duwarah SG, Barman H, Hoque R, Mili D, et al.Characteristics of Paediatric Scrub Typhus during an Outbreak in the North Eastern Region of India: Peculiarities in Clinical Presentation, Laboratory Findings and Complications. Indian J Paediatr. 2011;78:1365-70. DOI: doi.org/10.1007/s12098-011-0470-5

2. Gurung S, Pradhan J, Bhutia PY. Outbreak of scrub typhus in the North East Himalayan region-Sikkim: an emerging threat. Indian J Med Microbiol. 2013;31:72-4. DOI: 10.4103/0255-0857.108729

3. Jamil M, Lyngrah KG, Lyngdoh M, Hussain M. Clinical Manifestations and Complications of Scrub Typhus: A Hospital Based Study from North Eastern India. J Assoc Physicians India. 2014;62(12):19-23. PMID:2625941. 
4. Sarma N, Chakraborty S. Scrub typhus in Southern Districts of West Bengal. Indian J Dermatol. 2017;62(5):512. PMID: 28979015

5. Basu S, Saha A, Sarkar S, Sinha MK, Das MK, Datta R, et al. Clinical profile and therapeutic response of scrub typhus in children: A recent trend from Eastern India. J Trop Pediatr. 2019;65:139-46. DOI: doi.org/10.1093/tropej/ fmy027

6. Xu G, Walker DH, Jupiter D, Melby PC, Arcari CM. A review of the global epidemiology of scrub typhus. PLOS Negl Trop Dis. 2017;11(11):e0006062. PMID:29099844

7. Sengupta M, Anandan S, Daniel D, Prakash JA. Scrub typhus seroprevalence in healthy Indian population. J Clin Diagn Res. 2015;9(10):1. PMID: 26557523

8. Jakharia A, Borkakoty B, Biswas D, Yadav K, Mahanta J. Seroprevalence of scrub typhus infection in Arunachal Pradesh, India. Vector Borne Zoonotic Dis. 2016;16:659-63. PMID:27536803

9. Mørch K, Manoharan A, Chandy S, Chacko N, Alvarez-Uria G, Patil S, et al. Acute undifferentiated fever in India: a multicentre study of aetiology and diagnostic accuracy. BMC Infect. Dis. 2017;17(1):665. DOI: https://doi.org/ $10.1186 / \mathrm{s} 12879-017-2764-3$

10. Andrews MA, Ittyachen AM. Aetiology of acute febrile illness: a multicentre study from the province of Kerala in southern India. Trop Dr. 2018;48(4):322-5. DOI: doi.org/10.1177/0049475518794572

11. Mitra S, Gautam I, Jambugulam M, Abhilash KP, Jayaseeelan V. Clinical Score to Differentiate Scrub Typhus and Dengue: A Tool to Differentiate Scrub Typhus and Dengue. J Glob Infect Dis. 2017;9:12-17. DOI: 10.4103/0974-777X.199996

12. Mutyala SK, Mitra S, Abhilash KP, Jayaseelan V. Validation of a clinical score to differentiate scrub typhus and dengue. Curr Med Issues. 2017;15:121-4. DOI: 10.4103/cmi.cmi_18_17

13. Watt G, Jongsakul K, Chouriyagune C, Paris R. Differentiating dengue virus infection from scrub typhus in Thai adults with fever. Am J Trop Med Hyg. 2003;68(5):536-8. DOI: doi.org/10.4269/ajtmh.2003.68.536

14. Chang HC, Lin MC, Liu SF, Su MC, Fang WF, Chen YC, et al. The clinical differences between dengue and scrub typhus with acute respiratory failure in southern Taiwan. Infection. 2012;40(4):359-65. DOI: https://doi.org/ 10.1007/s15010-011-0239-9

15. Dhingra B, Sharma S, Mishra D, Kumari R, Pandey RM, Aggarwal S. Normal values of liver and spleen size by ultrasonography in Indian children. Indian Pediatr. 2010;47(6):487. DOI: https://doi.org/10.1007/ s13312-010-0090-6

16. Sundriyal D, Kumar N, Chandrasekharan A, Sharma B. Eschar: an important clue to diagnosis. Case Reports. 2013;bcr2013010105. DOI:10.1136/ bcr-2013-010105

17. Kundavaram AP, Jonathan AJ, Nathaniel SD, Varghese GM. Eschar in scrub typhus: a valuable clue to the diagnosis. J Postgrad Med. 2013;59(3):177-8. DOI: 10.4103/0022-3859.118033

18. Colby MF. Typhus and typhoid fever-calomel and the lancet. The Boston Medical and Surgical Journal as published by The New England Journal of Medicine.

19. Assawawiroonhakarn S, Geater A, Laoprasopwattana K. Using clinical profile and initiallaboratory results to differentiate leptospirosis, scrub typhus and dengue viral infections among children with an acute febrile illness. SE Asian J Trop Med. 2017;48(6):1274-80.

20. Elangovan B, Rajesh NT Clinical and laboratory predictors to differentiate severedengue from scrub typhus in children. Int J Contemp Pediatr. 2019;6:1223-7. DOI: http://dx.doi.org/10.18203/2349-3291.ijcp20192016

21. Adhikari S, Poudel RS, Shrestha S, Lamichhane P. Predictors of Mortality in ScrubTyphus Infection Requiring Intensive Care Admission in Tertiary Healthcare Centre ofNepal. Interdiscip Perspect Infect Dis. 2018:4867958. DOI: $10.1155 / 2018 / 4867958$.

22. Kim DM, Kim SW, Choi SH, Yun NR. Clinical and laboratory findings associated with severe scrub typhus. BMC Infect Dis. 2010;10:108. DOI: doi.org/10.1186/1471-2334-10-108

23. Sriwongpan P, Krittigamas P, Kantipong P, Kunyanone N, Patumanond J, Namwongprom S. Clinical indicators for severe prognosis of scrub typhus. Risk Manag Healthc Policy. 2013;6:43-9. DOI: 10.2147/RMHP.S52470.

24. Roy S, Datta Majumdar S, Chakrabartty S, Chakravarti S. A studyon scrub typhus in children: Experience in a tertiary care hospital. Sri Lanka J Child Health. 2019;48(2): 155-9. 